\title{
All-Optical Flip-Flop Based on an SOA/DFB-Laser Diode Optical Feedback Scheme
}

\author{
W. D’Oosterlinck, Student Member, IEEE, J. Buron, Student Member, IEEE, F. Öhman, \\ G. Morthier, Senior Member, IEEE, and R. Baets, Fellow, IEEE
}

\begin{abstract}
We report on the dynamic all-optical flip-flop (AOFF) operation of an optical feedback scheme consisting of a semiconductor optical amplifier (SOA) and a distributed feedback laser diode (DFB-LD), bidirectionally coupled to each other. The operation of the AOFF relies on the interplay between the optical powers in both the DFB-LD and the SOA. Switching times as low as $150 \mathrm{ps}$ for switch pulse energies of around $6 \mathrm{pJ}$ and a repetition rate of $500 \mathrm{MHz}$ have been measured. The contrast ratio was measured to be above $12 \mathrm{~dB}$.
\end{abstract}

Index Terms-Optical bistability, optical signal processing, semiconductor optical amplifiers (SOAs).

\section{INTRODUCTION}

A S THE network traffic load keeps on increasing, all-optical networks and more in particular packet or burst switched all-optical networks start to become a viable competitor to standard networks employing optical-electronic-optical conversions [1]. In these packet switched optical networks, there is a need for devices such as all-optical flip-flops (AOFFs) that show latching capabilities needed for packet header buffering and routing [2]-[4].

Over time different AOFFs have been proposed such as devices based on multimode interference bistable laser diodes [4], coupled laser diodes [5], or coupled Mach-Zehnder interferometers [6]. The reported switching times of these devices is typically a couple of hundred picosecond, while the length of the set and reset pulses used to switch the device are usually quite long.

Previously we reported static bistable operation of a semiconductor optical amplifier (SOA) bidirectionally coupled to a distributed feedback laser diode (DFB-LD) [7]. In addition to that, dynamic simulations showed that the device can also be used as an AOFF, i.e., can be set and reset using optical pulses [8]. Here we present for the first time experimental results on the dynamic behavior of this new AOFF. Switching times of about $150 \mathrm{ps}$, switch pulse energies of around $6 \mathrm{pJ}$, and a repetition rate of $500 \mathrm{MHz}$ were achieved with pulse lengths as low as 150 ps.

Manuscript received December 7, 2006; revised January 24, 2007. This work was supported in part by the European Network of Excellence ePIXnet. The work of W. D'Oosterlinck was supported by the Institute for the Promotion of Innovation through Science and Technology in Flanders (IWT-Vlaanderen) under a specialization grant.

W. D'Oosterlinck, G. Morthier, and R. Baets are with the Photonics Research Group, Department of Information Technology (INTEC), Ghent University-IMEC, 9000 Gent, Belgium (e-mail: wouter.doosterlinck@ intec.ugent.be).

J. Buron and F. Öhman are with the COM-DTU Department of Communications, Optics and Materials, Technical University of Denmark, $2800 \mathrm{Kgs}$. Lyngby, Denmark.

Digital Object Identifier 10.1109/LPT.2007.893756

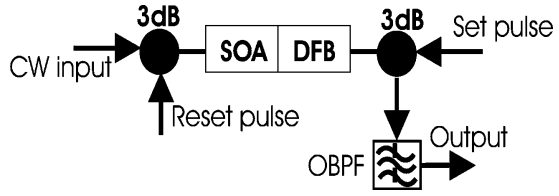

Fig. 1. Schematic representation of the AOFF.

\section{DEVICE DESCRIPTION}

The device used here as an AOFF basically consists of a travelling wave SOA which is bidirectionally connected to a laser diode (in this case a DFB-LD) through a coupler. In the actual device, the SOA is connected through a 1 by 4 coupler to four DFB laser diodes of which only one is used and shown here for clarity reasons. The bidirectional coupling causes a certain fraction of the DFB-LD power to be coupled into the SOA while at the same time the same fraction of the SOA output power gets coupled into the DFB-LD. This leads to a strong interaction between the optical fields of the DFB-LD and the SOA.

When a continuous-wave $(\mathrm{CW})$ beam is injected at the SOA side, it has been shown that bistable operation can occur with the actual state depending on the history of the device [7]. The two stable states are defined as either the state where the laser diode is switched ON or the state where the laser diode is switched OFF. It is pointed out that the DFB-LD could also be replaced by another antireflection-coated laser diode, e.g., a tunable sampled grating-distributed Bragg reflector laser diode.

In the ON-state, the laser light gets partially injected into the SOA causing the SOA to get deeper into saturation and thereby also decreasing the gain for the injected $\mathrm{CW}$ signal (and, therefore, also the power injected into the laser diode). When a reset pulse is added to the $\mathrm{CW}$ input power to the SOA (Port 1), the output power of the SOA rises and the laser diode can be switched OFF by this additional injected power. Once the pulse has disappeared again, the SOA has a higher gain for the $\mathrm{CW}$ signal than in the ON-state and the amplified CW signal can quench the gain in the laser diode forcing it to remain in the OFF-state. To switch back to the ON-state, a set pulse can be injected into the DFB-LD (Port 2 ), which, after having arrived at the SOA, can decrease the gain there momentarily giving the laser field the time to build up again.

A schematic representation of the device used as an AOFF is shown in Fig. 1. Since the device is based on optical feedback, the device can be used for a broad wavelength range of the CW input signal and set and reset pulses. The only limitation on the wavelength lies in the fact that reflections of the injected signal by the Bragg grating of the DFB laser diode need to be avoided. To that extent, the wavelength of the injected signals need to be sufficiently distant from the Bragg wavelength of the laser diode. 


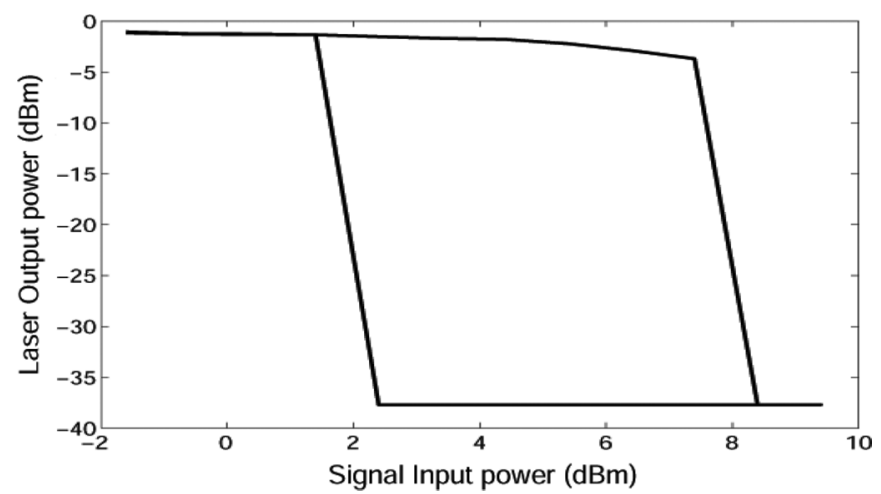

Fig. 2. Static response of the laser output power as a function of the input power to the SOA.

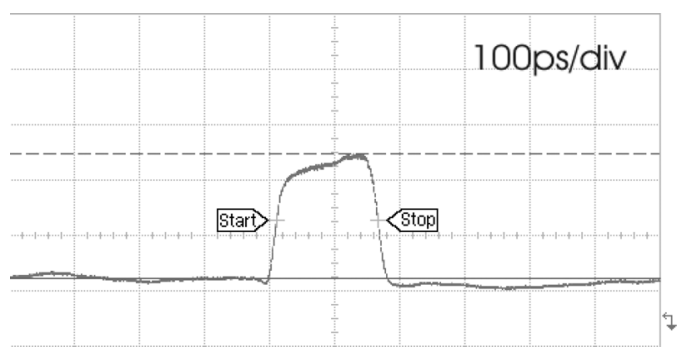

Fig. 3. Example of a set or reset pulse of about $150 \mathrm{ps}$ long.

\section{EXPERIMENTAL RESULTS}

To determine a proper working point for the AOFF, the static response of the device was first investigated. In Fig. 2, the output power of the DFB-LD as a function of the CW input power into the SOA is shown. It can be seen that for a $\mathrm{CW}$ input power between 2.4 and $8.4 \mathrm{dBm}$, the device exhibits bistability with a contrast ratio between the two states of over $35 \mathrm{~dB}$. The $\mathrm{CW}$ input power can be lowered by improving the fiber-to-chip coupling in future devices.

For the dynamic experiments, pulses as shown in Fig. 3 were generated using a 40-Gb/s modulator. From this pulse train, the set and reset pulses were obtained by using a 3-dB splitter. Both the set and reset pulses were then separately amplified by an erbium-doped fiber amplifier before being fed to the AOFF. The $\mathrm{CW}$ signal wavelength and the pulse wavelength were set at $1555 \mathrm{~nm}$. The wavelength of the DFB-LD was $1538.7 \mathrm{~nm}$. The drive current for the SOA was $103.5 \mathrm{~mA}$ and for the DFB-LD $101.4 \mathrm{~mA}$. The output state of the AOFF is determined by the output power of the DFB-LD (after passing through an optical bandpass filter).

AOFF operation using 150-ps-long set and reset pulses with energies of 6.1 and 4.4 pJ, respectively, is shown in Fig. 4. The $\mathrm{CW}$ power was $4.4 \mathrm{dBm}$ in this case. A contrast ratio of $12 \mathrm{~dB}$ can be observed. Here two set pulses (and also two reset pulses) are defined during each period of $6.4 \mathrm{~ns}$. The first pulse occurs at the beginning of the period while the second one is generated 2 ns later (as can be seen in Fig. 4). The delay between the set and reset pulse was determined by the difference in path length to the front (SOA) and backside (DFB) of the device and was measured to be about $1.2 \mathrm{~ns}$.

Looking in more detail at the actual switching of the device (Fig. 5), we note that a rise time of about 150 ps can be obtained.

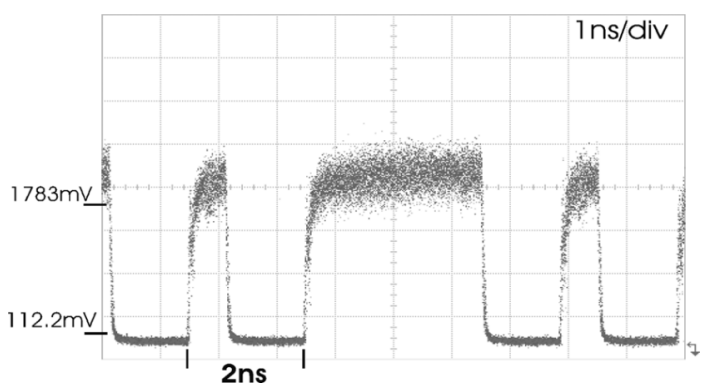

Fig. 4. Dynamic AOFF operation for a $\mathrm{CW}$ input power of $4.4 \mathrm{dBm}$ and using 150 -ps-long set and reset pulses.
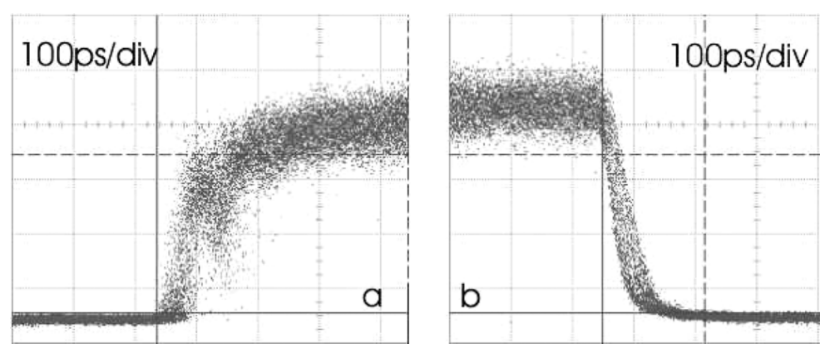

Fig. 5. Rise and fall time of the $\mathrm{AOFF}$ for a $\mathrm{CW}$ input power of $4.4 \mathrm{dBm}$ and 150 -ps-long set and reset pulses.

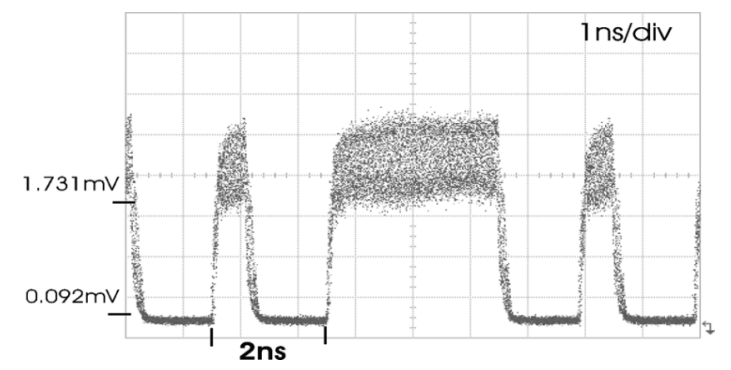

Fig. 6. Dynamic AOFF operation for a $\mathrm{CW}$ input power of $4.4 \mathrm{dBm}$ and using 300-ps-long set and reset pulses.

The relaxation oscillation of the laser diode can be observed as well along the rising edge. The fall time is also measured to be around $150 \mathrm{ps}$. The rise and fall times of the device suggest that the flip-flop can be turned OFF and ON again in just 300 ps. The shortest realizable $\mathrm{ON}-\mathrm{OFF}-\mathrm{ON}$ cycle may be significantly longer for the flip-flop to remain bistable. The repetition rate measures the actually realized cycle switching rate. Here we report a cycle repetition rate of $500 \mathrm{MHz}$.

When using 300-ps-long pulses, for the same CW input power, switching between the two states of the AOFF can still be obtained as shown in Fig. 6. In this case, the required switching energies were $5.5 \mathrm{pJ}$ for the set pulse and $7.5 \mathrm{pJ}$ for the reset pulse. Again a repetition rate of $500 \mathrm{MHz}$ between two set or reset pulses can be observed. The contrast ratio is in this case $12.7 \mathrm{~dB}$.

When looking at Fig. 7, one can see that due to the longer pulses both the rise and fall time increase. The rise time is around $200 \mathrm{ps}$ but the fall time increases significantly to about 250 ps. This might be due to the fact that the reset pulse first passes through the SOA, pushing it deeper into saturation, resulting in only a small initial change in the laser power that gets coupled back to the SOA. This small decrease of the laser power in turn causes only a small increase in the gain of the 

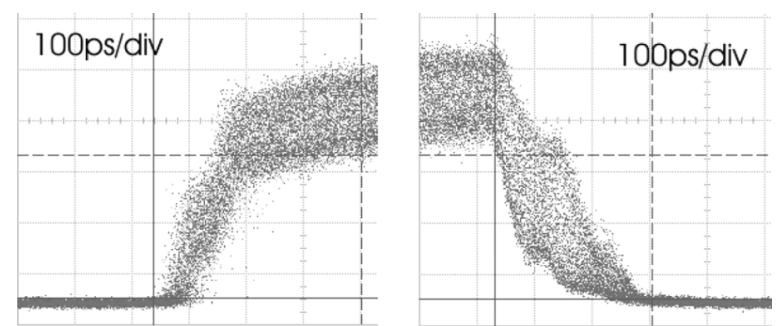

Fig. 7. Rise and fall time of the AOFF for a $\mathrm{CW}$ input power of $4.4 \mathrm{dBm}$ and 300-ps-long set and reset pulses.

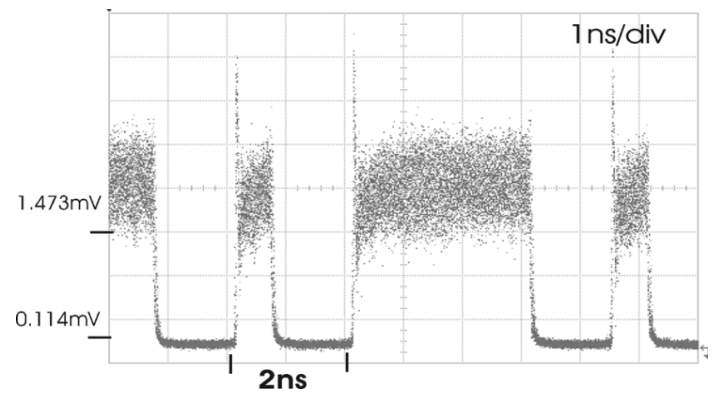

Fig. 8. Dynamic AOFF operation for a $\mathrm{CW}$ input power of $7.4 \mathrm{dBm}$ and using 150-ps-long set and reset pulses.
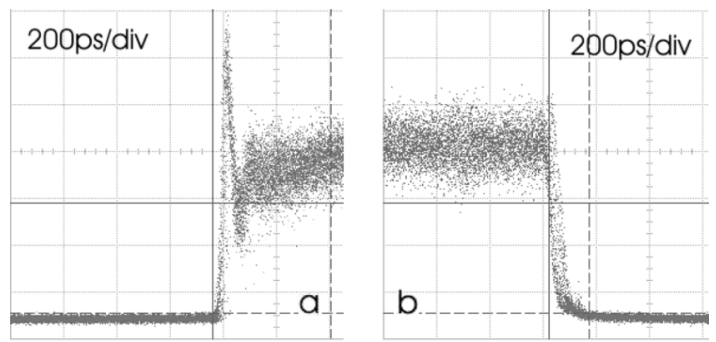

Fig. 9. Rise and fall time of the $\mathrm{AOFF}$ for a $\mathrm{CW}$ input power of $7.4 \mathrm{dBm}$ and 150 -ps-long set and reset pulses.

SOA. This optical feedback may last as long as the reset pulse is present in the device.

By changing the $\mathrm{CW}$ input power to the $\mathrm{SOA}$ to $7.4 \mathrm{dBm}$, it is shown in Fig. 8 that AOFF operation can be maintained over almost the entire range of $\mathrm{CW}$ powers for which bistability is obtained. The required pulse energies are in this case 15.8 and $4.3 \mathrm{pJ}$ for the set and reset pulses, respectively. The contrast ratio is now $11.1 \mathrm{~dB}$. The change in pulse energies as compared to the case where the $\mathrm{CW}$ input power to the SOA was $4.4 \mathrm{dBm}$ can be explained by looking at Fig. 2. For higher $\mathrm{CW}$ input powers, the working point of the device shifts to the right boundary of the bistable domain, implying that less additional power is needed to switch the AOFF from the ON-state to the OFF-state. On the other hand, since the working point moved away from the left boundary of the bistable domain, the power or energy needed to switch the AOFF from the OFF-state to the ON-state will be higher.

Fig. 9 again shows the switch ON and switch OFF response of the AOFF. In the turn-ON response of the AOFF [Fig. 9(a)], the relaxation oscillations of the DFB laser diode are more pronounced then in Fig. 5(a). This is caused by the fact that the higher $\mathrm{CW}$ input power $(7.4 \mathrm{dBm}$ in stead of $4.4 \mathrm{dBm})$ quenched the gain of the laser more in this case, giving rise to the increased height of the relaxation oscillations. However, both rise and fall time are again measured to be around $150 \mathrm{ps}$.

\section{CONCLUSION}

AOFF operation of an optical feedback scheme consisting of an SOA and a DFB-LD has been reported. Pulse energies as low as 4.4 and $6.1 \mathrm{pJ}$ for the set and reset pulse, respectively, have been shown. The AOFF shows a contrast ratio of $12 \mathrm{~dB}$ and can be operated with a pulse repetition rate of at least $500 \mathrm{MHz}$. Furthermore, it has been shown that both the $\mathrm{CW}$ input power and the pulse length can be varied, but increasing the pulse length also increases the switching time of the device. As the switch $\mathrm{ON}$ of the device essentially requires the laser diode to switch ON, the laser diode will impose a lower value to the obtainable switching times but by using short pulses switching times below 100 ps should be possible.

\section{REFERENCES}

[1] R. V. Caenegem, D. Colle, M. Pickavet, P. Demeester, F. Ramos, and J. Marti, "From ip over wdm to all-optical packet switching: Economical view," J. Lightw. Technol., vol. 24, no. 4, pp. 1638-1646, Apr. 2006.

[2] F. Ramos, E. Kehayas, J. Martinez, R. Clavero, J. Marti, L. Stampoulidis, D. Tsiokis, H. Avramopoulos, J. Zhang, P. Holm-Nielsen, N. Chi, P. Jeppesen, N. Yan, I. T. Monroy, A. Koonen, M. Hill, Y. Liu, H. Dorren, R. V. Caenegem, D. Colle, M. Pickavet, and B. Riposati, "Ist-lasagne: Towards all-optical label swapping employing optical logical gates and optical flip-flops," J. Lightw. Technol., vol. 23, no. 10, pp. 2993-3011, Oct. 2005.

[3] E. Kehayas, J. Seoane, Y. Liu, J. M. Martinez, J. Herrera, P. V. HolmNielsen, S. Zhang, R. McDougall, G. Maxwell, F. Ramos, J. Marti, H. J. S. Dorren, P. Jeppesen, and H. Avramopoulos, "All-optical network subsystems using integrated SOA-based optical gates and flip-flops for label-swapped networks," IEEE Photon. Technol. Lett., vol. 18, no. 16, pp. 1750-1752, Aug. 15, 2006.

[4] M. Takenaka, K. Takeda, Y. Kanema, Y. Nakano, M. Raburn, and T. Miyahara, "All-optical switching of $40 \mathrm{~Gb} / \mathrm{s}$ packets by mmi-bld optical label memory," Opt. Express, vol. 14, pp. 10 785-10 789, Oct. 2006.

[5] M. T. Hill, H. de Waardt, G. D. Khoe, and H. J. S. Dorren, "All-optical flip-flop based on coupled laser diodes," IEEE J. Quantum Electron., vol. 37, no. 3, pp. 405-413, Mar. 2001.

[6] M. T. Hill, H. J. S. Dorren, X. J. M. Leijtens, J. H. den Besten, T. de Vries, J. H. C. van Zantvoort, E. Smalbrugge, Y. S. Oei, J. J. M. Binsma, G. D. Khoe, and M. K. Smit, "Coupled mach-zehnder interferometer memory element," Opt. Lett., vol. 30, pp. 1710-1712, Jul. 2005.

[7] W. D'Oosterlinck, G. Morthier, R. Baets, and T. Erneux, "Optical bistability in a traveling-wave SOA connected to a dfb laser diode: Theory and experiment," IEEE J. Quantum Electron., vol. 42, no. 8, pp. 739-746, Aug. 2006.

[8] W. D'Oosterlinck, G. Morthier, T. Erneux, and R. Baets, "Simulation of the switching of an all-optical flip-flop based on a SOA/DFBlaser diode optical feedback scheme," in 2006 Int. Conf. Photonics in Switching, Heraklion, Crete, Greece, Paper 0.1.3. 